\title{
Olanzapine in practice: a prospective naturalistic study
}

\author{
David Taylor, Shameem Mir and Shubra Mace
}

\begin{abstract}
Aims and method The study aimed to evaluate the effectiveness of the naturalistic use of olanzapine. Prescribers of olanzapine were asked to provide baseline and six-week Brief Psychiatric Rating Scale scores for 56 in-patients. Withdrawals from treatment were also noted.

Results Olanzapine was not effective in any of the 12 patients with refractory schizophrenia and four patients worsened. In 36 patients with non-refractory schizophrenia, 16 (44\%) improved and 10 (28\%) were categorised as treatment failures. Of eight patients with non-schizophrenic psychosis, only one improved and two were treatment failures.

Clinical implications Olanzapine is effective in treating non-refractory schizophrenia, but appears to have no beneficial effect in refractory schizophrenia.
\end{abstract}

Olanzapine is an atypical antipsychotic which was introduced in Europe in 1996. Its advantages over typical drugs include a very low propensity to cause extrapyramidal sideeffects (Beasley et al, 1996) and improved efficacy against negative symptoms (Tollefson et al, 1996). Olanzapine closely resembles clozapine in chemical structure and in in vitro pharmacology (Kerwin \& Taylor, 1996) and, like clozapine, shows relatively low in vivo $\mathrm{D}_{2}$ receptor occupancy (Pilowsky et al, 1996). Unlike clozapine, olanzapine has not been shown unequivocally to be effective in refractory schizophrenia.

The effectiveness of olanzapine in refractory schizophrenia remains largely unknown. In addition. relatively little is known of the effects of olanzapine in a naturalistic setting, that is, outside the artificial setting of a clinical trial. Accordingly, we sought to evaluate the use of olanzapine in patients with psychotic illness, including refractory schizophrenia, in a natural, uncontrolled clinical setting.

\section{The study}

The study took place in the Bethlem and Maudsley NHS Trust in London. This hospital trust mainly treats patients from its inner-city catchment area, but a minority of patients are tertiary referrals from elsewhere in the UK.

The sample comprised in-patients who were consecutively prescribed olanzapine between October 1996 and October 1997. Patient consent was not obtained because the study did not alter normal practice. Prescribers of olanzapine were contacted and asked to provide the following information: patient name, age, gender and the reason for prescribing olanzapine. Prescribers were also asked to evaluate the patient's clinical condition using the 18-item Brief Psychiatric Rating Scale (BPRS; Overall \& Gorham, 1962), scored $1-7$, to provide a baseline BPRS score before starting olanzapine.

Six weeks later, prescribers were again contacted and requested to provide the patient's BPRS (at six weeks) or the reason for olanzapine withdrawal (where applicable). Prescribers were familiar with the use of the BPRS and ratings were performed by the same prescriber for each patient.

Information on previous antipsychotic therapy and on ICD-10 (World Health Organization. 1992) diagnosis was obtained by a retrospective review of case notes. All other patient data were checked for accuracy at this stage.

Collected data were divided into three groups for analysis according to the following criteria.

(a) Treatment refractory schizophrenia

Prior unsuccessful treatment with three different antipsychotics at effective treatment doses (>15 mg haloperidol or equivalent) for at least six weeks each; and BPRS score of $\mathbf{4 5}$ or more.

\section{Non-refractory}

BPRS score of 44 or less and/or previous therapy not meeting criteria for treatment-refractory'.

(b) Related psychoses

Patients not diagnosed as suffering schizophrenia according to ICD-10 guidelines. 
Table 1. Summary of patient characteristics and outcomes

\begin{tabular}{|c|c|c|c|}
\hline n (female:male) & $\begin{array}{l}\text { Treatment refractory } \\
\text { schizophrenia } \\
12(8: 4)\end{array}$ & $\begin{array}{l}\text { Non-treatment } \\
\text { refractory schizophrenia } \\
36(15: 21)\end{array}$ & $\begin{array}{l}\text { Non-schizophrenic } \\
\text { psychosis } \\
8(1: 7)\end{array}$ \\
\hline \multicolumn{4}{|l|}{ Diagnosis } \\
\hline Schizophrenia & 10 & 34 & \\
\hline Schizo-affective disorder & 2 & 2 & \\
\hline Unspecified non-organic psychosis & & & 2 \\
\hline Personallity disorder & & & $\overline{1}$ \\
\hline Learning disabillties & & & 1 \\
\hline Delusional disorder & & & 1 \\
\hline $\begin{array}{l}\text { Predominantly obsessional } \\
\text { thoughts }\end{array}$ & & & 1 \\
\hline No clear ICD-10 diagnosis & & & 2 \\
\hline Mean age (range) & $40.8(21-72)$ & $45.4(22-91)$ & $33.8(20-60)$ \\
\hline Mean baseline BPRS (range) & $53.1(45-65)$ & $38.8(22-79)$ & $42.9(30-65)$ \\
\hline Mean BPRS 6-week (range) & $50.7(40-87)^{\prime}$ & $31.2(19-61)^{2}$ & $40.3(23-56)$ \\
\hline \multicolumn{4}{|l|}{ Drug switched from } \\
\hline Typical oral & 3 & 12 & 1 \\
\hline Depot & 3 & 11 & 1 \\
\hline Risperidone & 4 & 18 & 2 \\
\hline Sertindole & 1 & & 1 \\
\hline Clozapine & 1 & & 3 \\
\hline \multicolumn{4}{|l|}{ Outcome } \\
\hline Improved (\%) & $0(0 \%)$ & $16(44 \%)$ & $1(12 \%)$ \\
\hline Same (\%) & $8(66 \%)$ & $10(28 \%)$ & $5(63 \%)$ \\
\hline Treatment failure (\%) & $4(33 \%)$ & $10(28 \%)$ & $2(25 \%)$ \\
\hline
\end{tabular}

BPRS, Brief Psychiatric Rating Scale.

1. Treatment withdrawn before six weeks in three patients: for analysis $n=9$ at six weeks

2. Treatment withdrawn before six weeks in five patients: for analysis $n=31$ at six weeks.

Data were collected for all patients beginning olanzapine between October 1996 and October 1997. Prescribers were allowed to adjust the dose of olanzapine as they wished. They were encouraged to withdraw previously prescribed antipsychotic therapy before or during the sixweek study period, according to their usual practice.

Efficacy was evaluated by categorising patients as 'improved' $(\geqslant 20 \%$ fall in BPRS score and a final score of 35 or less): 'treatment failure' $(\geqslant 20 \%$ rise in BPRS score or withdrawal from therapy); or 'same' (change in BPRS score $<20 \%$ or larger fall with final score of 36 or more). For the purpose of this efficacy analysis patients were evaluated in the three identified groups (as already described).

\section{Findings}

A total of 56 patients were prescribed olanzapine. Of these. 48 had firm ICD-10 diagnoses of schizophrenia, of whom 12 were categorised as treatment refractory. Eight patients were not clearly diagnosed as suffering from schizophrenia. Table 1 provides a summary of patient demographic data and patient outcomes.

\section{Comments}

This open, naturalistic study provides an interesting insight into the use of olanzapine in the treatment of psychosis in clinical practice. Olanzapine was found not to be effective in treating carefully defined refractory schizophrenia: not one of 12 patients improved and four were classified as treatment fallures. In non-refractory schizophrenia, olanzapine was, unsurprisingly, found to be much more effective, with $44 \%$ of patients showing improvement and only $28 \%$ defined as treatment failures. Olanzapine was only effective in one of 12 patients with related psychoses, although the small number of patients and the diversity of diagnoses make difficult any meaningful interpretation.

Our observations of the poor efflcacy of olanzapine in refractory schizophrenia are in contrast to those of other workers (Martin et al. 1997: Baldacchino et al, 1998) who have found that olanzapine, in similar naturalistic studies, is effective in a substantial proportion of patients with refractory illness. The most likely explanation for this disparity is that our criteria for refractoriness and clinical improvement were 
more stringent. Ours were largely based on the seminal study of Kane et al (1996) who showed that $30 \%$ of refractory patients who were given clozapine responded over six weeks. Martin et al (1997) demonstrated that $36 \%$ of patients responded to olanzapine, but in their study refractoriness was defined as that not responding to at least two (not three) antipsychotics given for four (not six) weeks. Patients were also less ill than those in our study: patients had baseline BPRS scores of 24 or more (scored 0-6), equivalent to 42 when scored 1-7. Baldacchino et al (1998) used no recognised criteria to classify treatment refractoriness or clinical changes.

It is noteworthy that the most recent study of olanzapine in treatment refractory schizophrenia used "Kane criteria" and found olanzapine to be no more effective than chlorpromazine (Conley et al, 1998).

Further support for the observation that olanzapine was not effective in refractory schizophrenia comes from a brief analysis of those patients switched from clozapine to olanzapine in this study. Four were switched from clozapine, one showed no change and three were classified as treatment failures.

In the $\mathbf{3 6}$ patients with non-refractory schizophrenia, outcome was generally good. Forty-four per cent showed a worthwhile response and $28 \%$ remained clinically the same by our definition. Many of these patients had tolerated poorly other drugs. Indeed six of the 10 who remained clinically the same were said previously to have had problems with adverse effects. That these patients evidently managed to complete six weeks' treatment with olanzapine in a naturalistic setting is an important observation, despite their lack of improvement. Indeed, the total number of patients completing six-weeks' treatment with olanzapine (40 of 56) is an indirect testament to its tolerability.

Naturalistic studies, as this one, involve no randomisation of treatments and no comparator treatments. Assessors and prescribers are usually aware of the treatment being used. Thus, this study lacks the scientific rigour of a properly controlled clinical trial and so its results must be treated with some caution. Nevertheless, this study does reflect, to a large extent, normal practice in our unit and perhaps other similar hospitals. We made strenuous efforts not to interfere with normal practice, so that a proper, naturalistic evaluation could be made.

We recognise that the patient sample in this study was small and heterogenous, but nevertheless, believe that the results presented here are a useful and accurate reflection of practice and of treatment outcome with olanzapine. Olanzapine appears to be well-tolerated and is effective in many patients with non-refractory schizophrenia. In refractory schizophrenia, olanzapine proved ineffective.

\section{Acknowledgements}

We thank Professor R. Kerwin, Dr T. Sharma and all the prescribers involved in this study.

\section{References}

Baldacchino, A. M., Stubbs, J. H. \& Nevison-AndRews, D. (1998) The use of olanzapine in non-compliant or treatment-resistant clozapine populations in hospital. Pharmaceutical Joumal, 260, 207-209.

Beasley, C. M., SANger, T., SATterlee, W., et al (1996) Olanzapine versus placebo: results of a double blind. fixed dose olanzapine trial. Psychopharmacology, 124, 159-167.

Conley, R. R., TAMminga, C. A. BARTKo, J. J., et al (1998) Olanzapine compared with chlorpromazine in treatment-resistant schizophrenia. American Joumal of Psychiatry. 165, 914-920.

KANE, J., Hongfield, G., Singer, J., et al (1996) Clozapine for the treatment-resistant schizophrenic: a doubleblind comparison with chlorpromazine. Archives of General Psychiatry. 46. 789-796.

KERWIN, R. \& TAYLOR, D. (1996) New antipsychotics - a review of their current status and clinical potential. CNS Drugs, 6, 71-82.

MARTIN. J.. GOMEZ. J. C.. GARCIA-BERNARDO. E.. et al (1997) Olanzapine in treatment-refractory schizophrenia: results of an open-label study. Journal of Clinical Psychiatry. 88, 479-483.

OVERAL, J. E. \& GORHAM, D. R. (1962) The Brief Psychiatric Rating Scale. Psychological Reports, 10, 799-812.

PILowsky, L. S., Busatro, G. F., TAYLoR, M., et al (1996) Dopamine $D_{2}$ receptor occupany in vivo by the novel antipsychotic olanzapine: ${ }^{123}$ I IBZM single photon emission topography (SPET) study. Psychopharmacology, 124, 148-153.

Tollefson, G. D., Beasley, C. M., Tran, P. V., et al (1996) Olanzapine versus haloperidol in the treatment of schizophrenia and schizoaffective and schizophreniform disorders: results of an international collaborative trial. American Joumal of Psychiatry. 184, 457-465.

WORLD HEALTH ORGanization (1992) The Tenth Revision of the International Classification of Diseases and Related Health Problems (ICD-10). Geneva: WHO.

* David Taylor, Chief Pharmacist, Shameem Mir. Senior Clinical Pharmacist, and Shubra Mace, Clinical Pharmacist, Maudsley Hospital. Denmark Hill, London SE5 8AZ

*Correspondence 